\title{
Design of Internet of Things Business Model with Deep Learning Artificial Intelligence
}

\author{
Yong-kyu Lee ${ }^{1}$ and Dea-woo Park ${ }^{2 *}$ \\ ${ }^{1,2}$ Hoseo Graduate School of Venture, Hoseo University, Seoul, \\ (06724), South Korea \\ 1ceo@web-planner.net,.2*prof_pdw@naver.com
}

\begin{abstract}
The competition of Go between AlphaGo and Lee Sedol attracted global interest; AlphaGo was victorious. The core function of AlphaGo is a deep-learning system, in which the computer learns by itself. It is now said that the utility of deep-learning systems using artificial intelligence is verified. Recently, the government of South Korea passed the lnternet of Things (IoT) Act with a view to developing a business model to promote loT. In this paper, we identify IoT market sales through IoT market trend analysis and extract an IoT business model. Then, we apply the findings to Deep learning AI technology in order to design an internet business model for Deep learning AI. We look at Deep Learning as it is used in smart home technology, autonomous vehicles, and a healthcare wearable device. This paper will be fundamental for social development using the technologies of the 4th industry.
\end{abstract}

Keywords: IoT, Artificial Intelligence, Deep Learning, Business Model

\section{Introduction}

The competition of Go between AlphaGo and Lee Sedol attracted global interest; AlphaGo was victorious. The core function of AlphaGo is a deep-learning system, in which the computer learns by itself. It is now said that the utility of deep-learning systems using artificial intelligence [1] is verified. Deep learning technology involves an artificial neural network that imitates the human brain. There are two applications of this technology: one is recognition through deep learning, and the other is self-learning. Recently, a self-study technique has been developed which can make decisions. It recognizes not only simple text but also patterns such as images, voice and so on [2].

The vast amount of data being generated that forms the basis of machine learning is estimated by IBM to be about 2.5 quintillion bytes per day [3].

By 2020, the planet's population will reach 7.6 billion people. CISCO put out a statement claiming that the number of connections, generated by personal devices, between things and people will reach 25 billion [4].

We looked at a study which shows that the performance of a purchase prediction model can be improved by using deep learning to analyze the click stream data of an online bookstore [5].

Based on there being 7 Internet objects per person, society has a super-connected environment, and a huge amount of big data is being created. Recently, the South Korean government passed the IoT [6] Act and is developing a business model to promote IoT.

This study is concerned with analyzing the IoT business environment using deeplearning AI and designing specialized 4th industry business models.

Received (April 8, 2018), Review Result (June 5, 2018), Accepted (June 9, 2018)

${ }^{*}$ Corresponding Author 


\section{Related Works}

\subsection{IoT}

IoT can be viewed as a network infrastructure in which physical or virtual objects are interconnected, interacting without human intervention, and exchanging data and information [7]. The Ministry of Science and ICT defines the IoT business as "a collection of industry groups that collect and process data using IoT technology for business purposes." It is subdivided into base equipment industry, infrastructure support industry, and appliances industry [8].

\subsection{AI Deep Learning}

Deep learning has great strengths in the pattern recognition of big data. In 2045, it is predicted that artificial intelligence will be 1 billion times more powerful than human intelligence. Deep Face, Facebook's AI face recognition, is accurate to $97.25 \%$. This is close to human recognition rates, to the extent that there is a gap of only $0.25 \%$ compared with human recognition accuracy of $97.5 \%$.

Artificial Intelligence Deep Learning, based on Big Data, is capable of self-learning as it analyzes patterns, not only texts, but also images and sounds. This means that people will be able to predict and prescribe their actions.

\subsection{Business Model}

With the proliferation of Internet services and mobile Internet due to the spread of smartphones, a new business model is needed. A 2006 study by Krista M. Donaldson \& Kosuke Ishii \& Sheri D. Sheppard proposed a new tool for business customization; CVCA (Customer Value Chain Analysis) is a methodological tool used in product design which clearly defines stakeholder relationships in the lifecycle of a product or service.

\section{Analysis of IoT Business Status}

\subsection{Analysis of IoT Market Trend}

The IoT market has accelerated since Sohn Jung-eu (Masayoshi Son), the chairman of Softbank, acquired a British semiconductor company, ARM. ARM has invested in IoT, which connects everything from cars to light bulbs to the Internet, and also accounts for 90\% of the world's smartphone semiconductors. Hardware vendors such as Intel, Samsung Electronics, AMD and Apple are also in the spotlight, and MVNOs such as Verizon and SKT are also increasing their services. Service application vendors such as Google, Facebook, and Instagram are also accelerating towards the IoT market.

\subsection{Analysis of IoT Market Sales}

Table 1 shows the market sales trends of the Internet industry in Korea [9]. 
Table 1. Global Outlook of the loT Market

\begin{tabular}{|c|c|c|c|c|}
\hline \multicolumn{5}{|c|}{ Unit: \$ 100 million, } \\
\hline \multicolumn{2}{|r|}{ Item } & $2013 \mathrm{Y}$ & $2022 \mathrm{Y}$ & CAGR \% \\
\hline \multirow[t]{4}{*}{ Devices } & Chip sets & 58 & 281 & 19.2 \\
\hline & Modules & 102 & 477 & 18.7 \\
\hline & Terminals & 1,728 & 3,692 & 8.8 \\
\hline & Total & 1,888 & 4,450 & 10.0 \\
\hline \multirow{4}{*}{$\begin{array}{l}\text { Mobile } \\
\text { communication } \\
\text { networks }\end{array}$} & GSM/HSPA & 31 & 69 & 9.3 \\
\hline & CDMA & 42 & 78 & 7.1 \\
\hline & LTE & 14 & 201 & 34.5 \\
\hline & Total & 95 & 391 & 17.0 \\
\hline \multirow{5}{*}{$\begin{array}{l}\text { System } \\
\text { operators }\end{array}$} & Product manufacturers & 12 & 694 & 57.0 \\
\hline & Systems integrators & 14 & 1,436 & 67.3 \\
\hline & $\begin{array}{l}\text { Specific application rental } \\
\text { business operators }\end{array}$ & 8 & 904 & 69.1 \\
\hline & $\mathrm{B} 2 \mathrm{~B} / \mathrm{B} 2 \mathrm{C}$ service operators & 3 & 521 & 77.4 \\
\hline & Total & 37 & 3,555 & 66.1 \\
\hline \multirow{7}{*}{$\begin{array}{l}\text { Services and } \\
\text { applications }\end{array}$} & In-car telematics & 5 & 1492 & 88.4 \\
\hline & Fleet Management & 1 & 186 & 78.7 \\
\hline & Smart Grid and management & 2 & 215 & 68.2 \\
\hline & Fixed wireless communications & 1 & 271 & 86.4 \\
\hline & Household appliances & 1 & 1,184 & 119.5 \\
\hline & Other & 1 & 204 & 80.6 \\
\hline & Total & 11 & 3,552 & 90.0 \\
\hline \multicolumn{2}{|l|}{ Total } & 2,031 & 11,948 & 21.8 \\
\hline
\end{tabular}

The IoT market is diverse, including devices, mobile communications networks and systems businesses, services and applications. According to Machina Research's forecast for 2013, the global IoT market, which was still in the early stages of growth, was predicted to grow at an average annual rate of $21.8 \%$ from 2013 to 2022, reaching $\$ 1.19$ trillion.

Analysis of sales in the global IoT market shows that the handset business will grow strongly in the early stage and that the services and application market will grow strongly in the stabilization phase.

\subsection{Extract IoT business Model}

The growth rate of IoT is increasing every year by $21.8 \%$, and data is constantly being generated by objects connected to the internet. Deep learning AI learns from big data, not only text data but also handwriting, photographic images, voice, video and so on. 
The first thing that Deep Learning AI can do is to find out where the data you want is located. Secondly, it processes information acquired through learning and thereby creates new information. Thirdly, it can make predictions based on its learned information.

Deep Learning AI learns from big data generated by objects and creates a business model that can be applied to various fields.

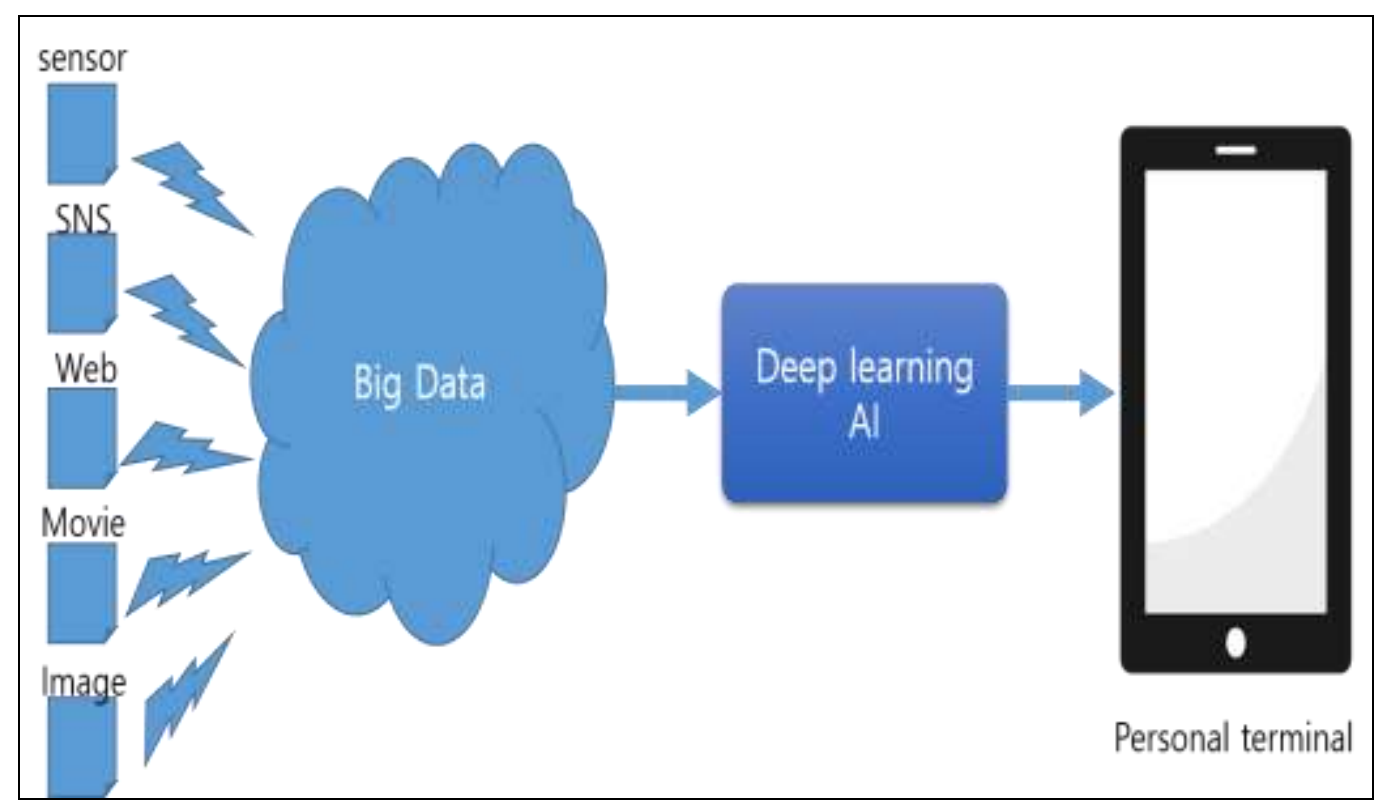

Figure 1. Extract loT Business Model

The most important data collection device for the Deep Learning AI system is the smartphone. Robots will eventually play a central role in the home and office. And when people are on the move, automobiles, subways, buses and so on will continuously collect data for the Deep Learning AI system.

Customers who use this information will become smarter in business. It has the advantage of using a smart phone to have enormous information power, and to increase autonomy to infinity. On the other hand, customers who are very picky and indifferent, with complicated demands, will become smart customers because of the huge amount of information that will be available.

The key factor in the IoT business model is the human factor. Therefore, Deep learning AI learns and develops an understanding of people in order to do business.

Everyone has different needs and wants regarding services and products. Customized service is required. A personalized future-predictive business model should be provided by learning about people in order to discover the information that the user wants, so as to offer and provide personalized services based on prediction of what the user will demand.

\section{Design IoT Business Model using Deep Learning Al}

\subsection{Applied Technology by Deep Learning AI}

Analyzing purchase patterns makes it possible to predict the behavior of a user. forecast-based AI services relating to offline as well as online behaviour will be developed.

Tapingo services in the US colleges provide services through analysis of these customer behavior patterns. When you order coffee or fast food at a certain time, it analyzes the relevant data and then automatically asks you "Do you want to drink coffee 
now? If you choose it now, you will be able to take a coffee in Starbucks in five minutes if you are walking at the current speed."

\subsection{Applying Deep Learning AI to the Internet Business Model}

Deep Learning AI collects data generated by objects with sensors, which is transmitted and stored in the cloud. Deep learning AI then generates new information, the purpose of which is to provide convenience to people who will use it.

We want to apply artificial intelligence by dividing the business model into three.

One is to apply AI to a residence, second, to a transportation vehicle, and finally to a wearable band device for monitoring health.

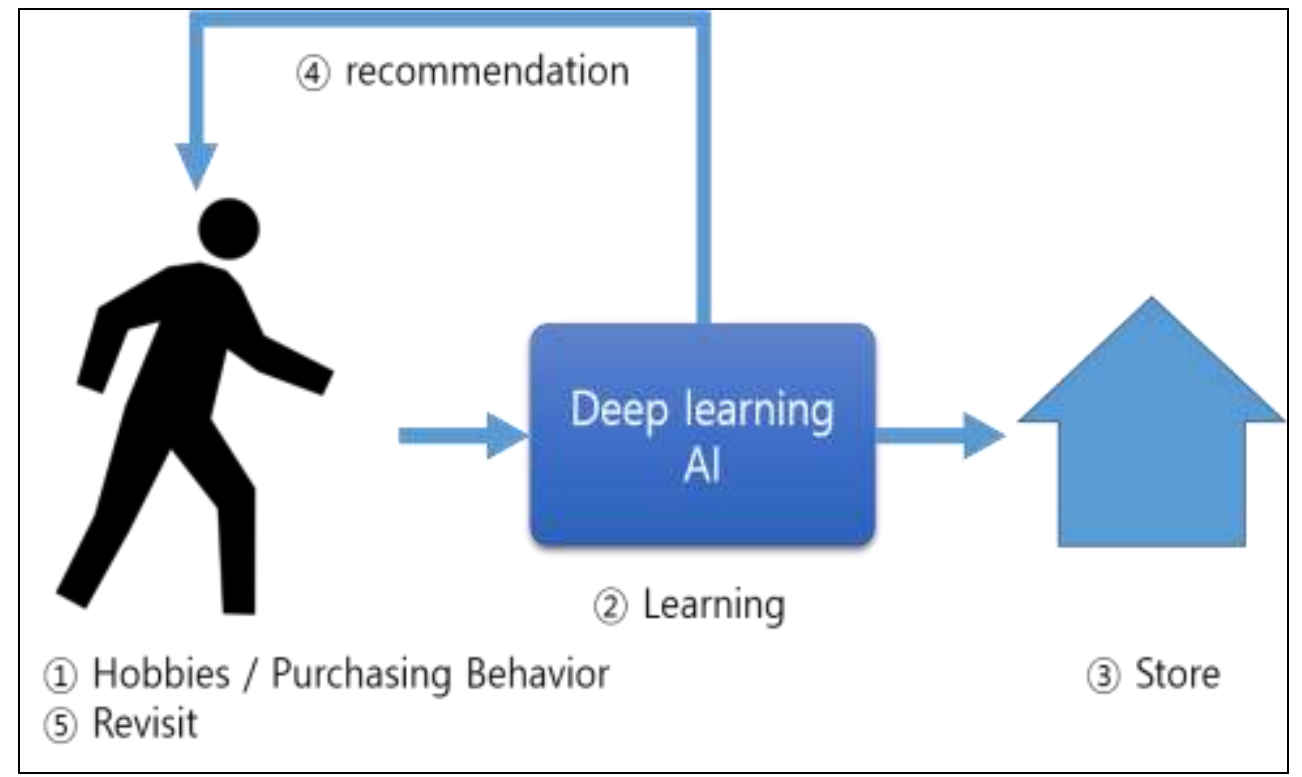

Figure 2. Applying Deep Learning Al to the Internet Business Model

4.2.1. Apply Deep Learning AI to Smart Home: Nest is a smart home device that automatically adjusts the temperature in the house. By analyzing manual temperature adjustments made by the owner, Machine Learning automatically adjusts to the optimal state. Thus thermostat and security devices can be included in smart home control, and not just home appliances.

The role of the smart home is as follows. When you leave your office, the correct temperature will be set, and lighting and music will be set when you arrive home. An electric rice cooker will activate and warm water will fill the tub in your bathroom. A robot vacuum cleaner will work automatically. The washing machine will be doing the laundry and sorting family members' clothes.

A company might control the IoT terminal, allowing the smart home to connect through its products. From the customer's point of view, the value of energy savings and convenience justifies the price of the AI terminal. Thus, a business model is established.

4.2.2. Deep-Running AI for Autonomous Vehicles: Google and Apple's autonomous cars are not traditional cars, but IoT devices. When deep learning AI is connected to an autonomous vehicle, you can spend a comfortable time driving and parking safely. All devices in the autonomous vehicle will be connected to the cloud to provide real-time optimization services.

The autonomous vehicle is connected to the smart home, connected to the Internet device in the house, and can play the role of the primary physician providing the health information of the family through the owner's health band. 
4.2.3. Apply Deep Learning AI to Healthcare Bands: Healthcare bands, which are wearable IoT devices, provide value by monitoring health states in comparison to the individual's optimal health status. Examples of states that can be monitored are number of steps walked per day, heart rate, blood pressure, glucose levels, stress index, and work rate.

If your physical condition is not good, you can contact your doctor and get a service that will provide your location.

\subsection{Deep Learning Using AI to Design an Internet Business Model}

The data generated by the smart home, the autonomous vehicle and the health care bands is uploaded by an internet node and processed by the deep learning AI application to create new information. AI allows people to live long, healthy, happy lives, and offers convenience and efficient use of time.

Deep Learning using artificial intelligence can create a new business model. We apply artificial intelligence in three sections. We will study the smart home intelligence business model, the business model for autonomous vehicle using AI and the business model for health care bands for monitoring human health.

4.3.1. Deep-Running AI Model Designed for Home: The value that a person feels at home is comfort and rest. To realize this value, AI analyzes the behavior pattern of each person living in the house and provides information and services satisfying their requirements.

When arriving home, your favorite comfortable temperature is set and the lighting is adjusted with colors that match your mood. The music you enjoy plays, and the news or important things of the day are announced. All these things are related to AI Deep Learning.

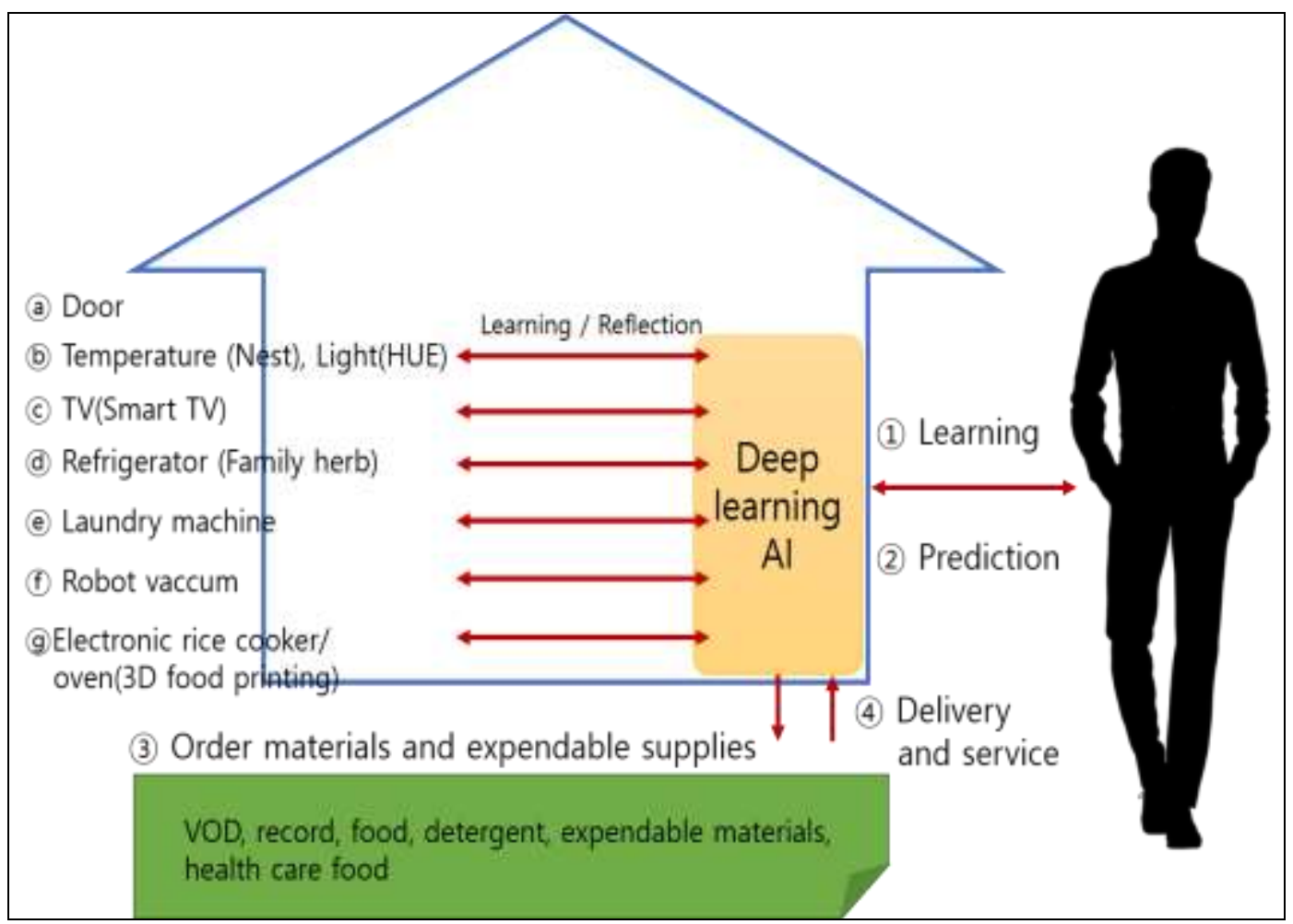

Figure 3. Deep-Running Al Model Designed for Home 
(1) Learning target: The items that are learned from an individual at home are as follows.

(a) Gate: The gate analyzes whether or not the person is a house member. If he / she is not living in the house, the system offers the visitor's information to the house owner to help initiate conversation. For those who have gained access illegally, it asks the police for help or shares images. If the person is a resident, it opens the house and informs them who is in the house. Then it analyzes aspects of the personality (facial expression, mood state, biorhythm, etc.,) and transmits this information to other devices in the house.

(b) Temperature, lighting: It pre-learns the temperature and lighting that each individual likes and adjusts the temperature according to the characteristic of the person in advance. After exercise, you can control cool and warm air according as the body temperature is high or low. Color and brightness of lighting are adjusted according to the individual's mood.

(c) TV (Smart TV): It sets TV programs according to the preference of the individual and records frequently viewed programs. And it provides information about people and products from broadcasting channels and aids in purchasing. In addition, it connects to smartphones for viewing on the smartphone or displays favorite images and screens on the TV. It performs the role of personal secretary by executing a service that summarizes the latest trends and news by selecting the kinds of information preferred by the individual.

(d) Refrigerator (family groceries): It provides information about the distribution periods of foods. It also orders food according to the consumed state of the products contained in the refrigerator and proposes recipes that can be prepared using the food inside the refrigerator, according to the individual's health condition.

(e) Washing machine: It washes family clothes and dries them individually, organizes clothes per individual, and orders detergent automatically.

(f) Robot Vacuum Cleaner: It cleans the house when it needs to be cleaned and arranges items in a personalized manner. Orders consumables when things are consumed or missing.

(g) Electronic rice cooker/oven (3D food printing): It cooks according to the time when an visual enters the house. These days, new types of fashionable dishes are made using 3D printing, or cooked in a microwave oven or electric oven.

(2) Prediction (prediction by learning customer behavior patterns): It predicts individual behavior through information gathered through each sensor for personal convenience.

(3) Order materials and consumables: It automatically orders items required for each step and confirms the order to complete payment.

(4) Delivery and service (VOD, recording, food, detergent, consumables, health food, etc.): Various kinds of necessities such as household necessities, groceries and consumables are ordered and delivered from various online and offline marts. If there is no one in the house, for safe delivery, it opens the door after checking and receives delivered items.

Demand is increasing for home appliances that are used in smart homes equipped with IoT functionality. Supplies and consumables which are continuously used in such appliances lead to continual customer purchases. This makes a business model. 
4.3.2. Deep-Running AI Model Design for Autonomous Vehicles: While driving a car, performing other tasks is difficult, and increases risk. When autonomous vehicles are deployed, they provide a space in which you can work and relax. While traveling on autonomous vehicles, AI predicts user's preferences and recommends restaurants. Artificial intelligence that recommends restaurants can receive financial commissions from the restaurants. This is the business model.

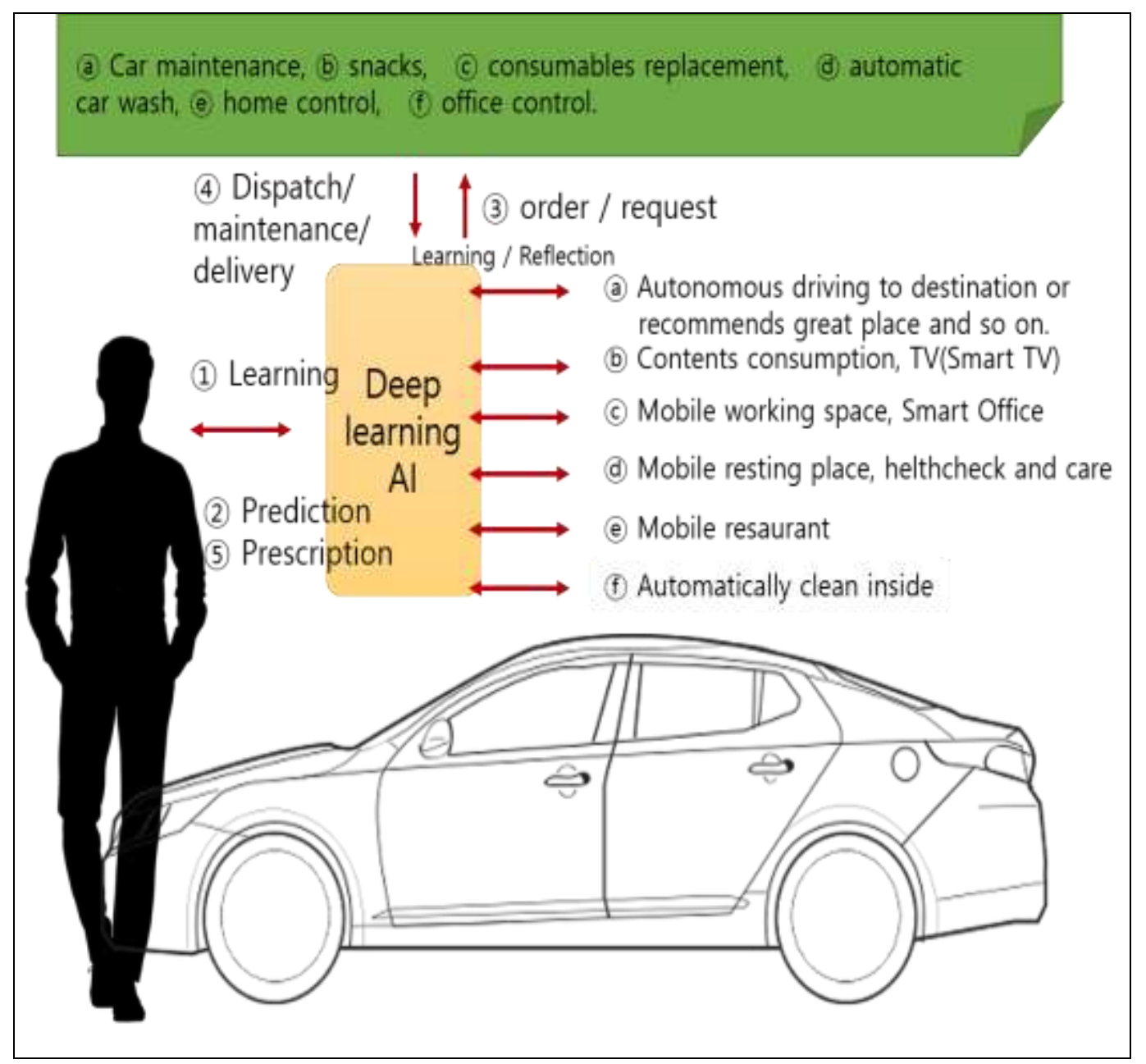

Figure 4. Deep-Running Al Model Design for Autonomous Vehicles

(1) Learning target: The content of learning of autonomous driving car is as follows.

(a) Autonomous driving / destination recommendation: It sets the destination according to the schedule of the individual and sets the shortest distance to get to the destination.

(b) Content consumption, TV (Smart TV) : You can watch programs that you couldn't finish watching at home in a self-driving vehicle while you are on the move, or you can watch news and up-to-date information tailored to your interests. It consumes or produces Game and SNS contents. In addition, you can consume tailored content or simulates.

(c) Mobile work space, Smart Office: In the case of a business meeting, it is possible to check e-mail and details of conversations that are shared with business partners, and to conduct meetings efficiently through rehearsal. In addition, a remote video conference can be carried out in an autonomous vehicle. If the individual is 
travelling, it can check the recommended course for trips at the destination and reviews of restaurant recommendations.

(d) Mobile resting space, health check and care: In a moving space, you can sleep in a resting area. Or you can obtain prescriptions through tele-medicine for personal health. Also, psychological therapy for stress management, and massage for physical health helps manage your wellbeing.

(e) Mobile dining space: When you move, you can place an order remotely before arriving at the restaurant. Food can be delivered by drone to your autonomous vehicle, saving time and space.

(f) Clean inside a car automatically with a robot cleaner: It makes the space fresh in the autonomous vehicle as the autonomous robot or autonomous vehicle cleans itself.

(2) Prediction: You can find optimal directions to your destination, or work while the car drives itself. Also, you can rest and examine your body to generate health predictions.

(3) Order / Request: Order various contents, goods, etc. in the autonomous vehicle.

(4) Dispatch / maintenance / delivery

(a) Vehicle maintenance: If a problem is found during self-diagnosis, it asks for vehicle maintenance and repair, or makes a reservation at a nearby repair shop. The vehicle can drive itself to the repair shop.

(b) Snacks: It orders snacks appropriate to your taste. It can also prepare snacks according to your schedule and health condition.

(C) Replace consumables: Self-diagnosis informs you of the condition of wear of consumables, and can order replacements. It tells you how to replace consumables on your smartphone or on the screen inside the car.

(d) Automatic car wash: the autonomous vehicle performs a car wash by itself, or visits a nearby car wash before returning home.

(e) Home control: when the autonomous vehicle arrives at the house, it adjusts the state of the house according to the characteristics of the individual. It sets the temperature of the house, prepares the meal, turns on the TV and plays music.

(f) Office control: When the autonomous vehicle arrives at the office, it adjusts the office environment according to the day's schedule. It turns on the computer, makes coffee, displays the work schedule, lists tasks in order of importance, and offers documents and presentation materials needed in a meeting.

(5) Prediction: The autonomous vehicle learns about the driver and predicts various conditions to meet the individual's requirements.

Demand for internet devices and content consumed in autonomous vehicles is increasing. It is also possible to have a business model that provides automatic order delivery or management services in conjunction with autonomous vehicle consumables.

4.3.3. Deep-Running AI Model Design for Healthcare Bands: Health is a high priority for people who want to live healthily and happily. By using wearable health care bands with deep running artificial intelligence, a customer's health status is monitored. Customized care and prescriptions will maintain a customer in good health. 


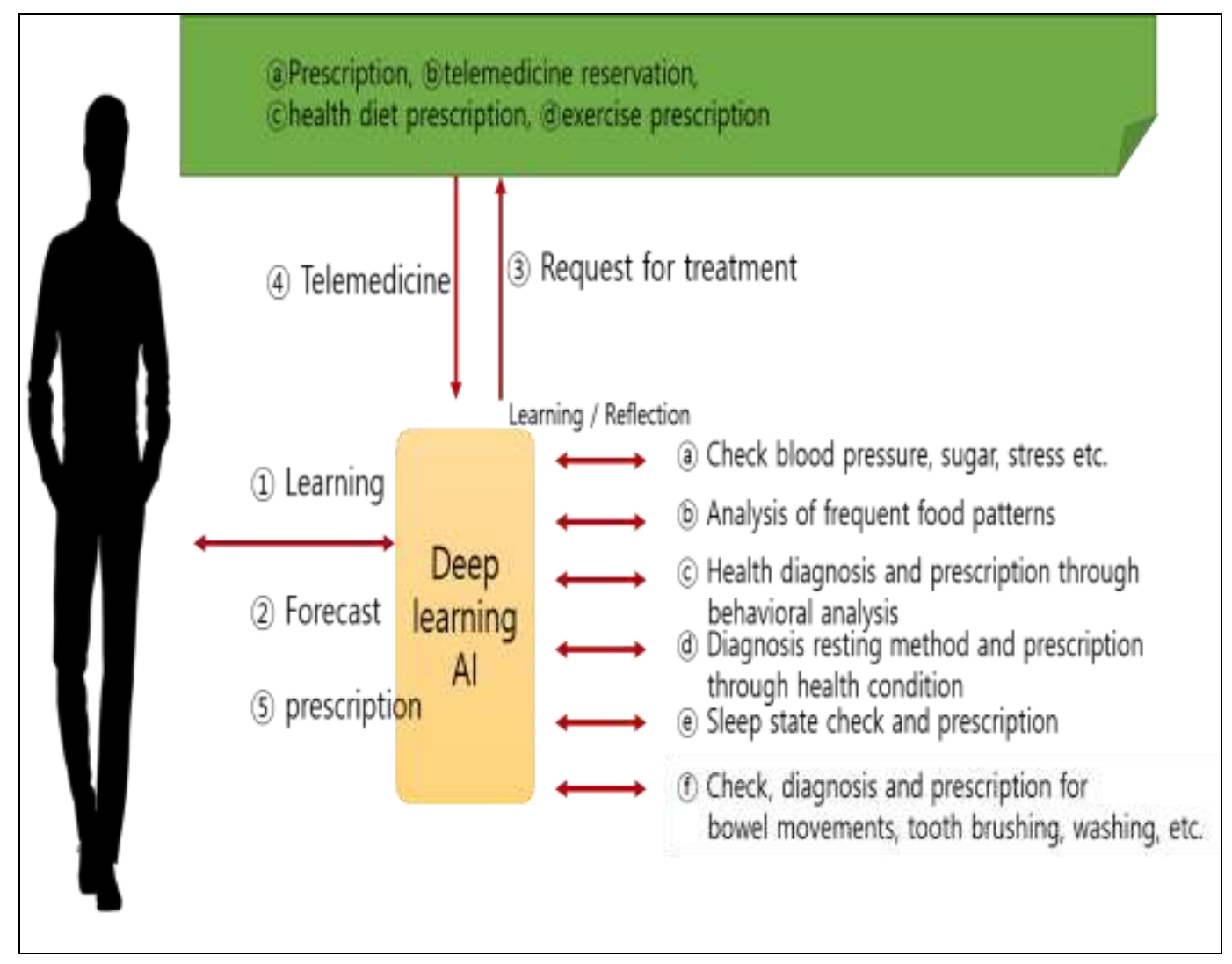

Figure 5. Deep-Running Al Model Design for Healthcare Bands

(1) Learning target: Healthcare wearables produce data to improve and maintain an individual's health.

(a) Check blood pressure, diabetes, stress, etc.

(b) Analysis of frequent food patterns

(C) Health diagnosis and prescription through behavioral analysis

(d) Resting methods and prescription according to health condition

(e) Sleep state check and prescription

(f) Check, diagnosis and prescription for bowel movements, tooth brushing, washing, etc.

(2) Forecast

(3) Request for treatment / Request for alternative treatment

(4) Telemedicine

(a) Prescription, (b)telemedicine reservation, (Chealth diet prescription, (d)exercise prescription

(5) prescription

Demand for wearable healthcare bands that can measure people's health status will increase. Demand for healthcare band services and consumables will increase, too. Customized health products, diagnostics and prescriptions will be needed. Here, business models for health-related products for clothing, food and housing will be possible. 


\section{Conclusion}

AI technology applying deep learning technology does not simplify the connection of IoT terminals, but it reveals organic connections between peoples, between things, and between people and things.

Business activities can now take place in a variety of areas through AI and IoT using new deep learning technology.

We can engage in business activities by developing technology and services that operate an artificial intelligence platform applying deep learning technology.

In this paper, we analyzed the IoT business environment using Deep Learning AI. Also, we designed a specialized business model through Deep Learning AI model design for IoT, autonomous vehicle, and health care bands using Deep Learning AI.

In the future, by using big data from IoT terminals, deep study of business models should be followed in order to apply learned results to new fields. In-depth study of the Deep Learning AI business model and on-the-ground empirical studies are needed to demonstrate the value and technology of the Deep Learning AI business model.

\section{References}

[1] D. Le Nguyen and M-E Lee, "OSEK/VDX Porting to the Teo-Wheel Mobile Robot Based on the Differential Drive Method", Journal of Information and Communication Convergence Engineering, vol. 10, no. 4, (2012) December, pp. 372-377.

[2] Y. Bengio, A. Courville and P. Vincent, "Representation learning: A review and new perspectives", IEEE Transactions on Pattern Analysis and Machine Intelligence, vol. 35, no. 8, (2013), pp. 1798-1828.

[3] Bringing big data to the enterprise, IBM 2012, Bringing big data to the enterprise. [Internet]. Available: http://www-01.ibm.com/software/sg/data/bigdata logged on (2015) November.

[4] Cisco IBSG, "Microsoft Word - IoT_IBSG_0411FINAL", (2011).

[5] J. W. Jong, S. Y. Han, H. J. Lee and J. H. Oh, "A Study of Predicting Consumer's Purchase Intention Using Clickstream Data for On-line Recommendation Services", (2012).

[6] S. Husain, A. Prasad, A. Kunz, A. Parageorgiou and J. Song, "Recent trends in standards related to the Internet of Things and Machine-to-Machine communications", Journal of Information and Communication Convergence Engineering, vol. 12, no. 4, (2014) December, pp. 228-236.

[7] C. S. Pyo, H. Y. Kang, N. S. Kim and H. C. Bang, "IoT (M2M) Technology Trends and Prospects", Journal of ICT, vol. 30, no. 8, (2013), pp. 3-10.

[8] B. G. Lee, G. C. Park and Ministry of Science, "ICT \& Future Planning. Survey and market analysis of IoT(Internet of Things)", Jinhan M\&B, (2014), pp. 91-94.

[9] D. Y. Joo, "Hyper Connected Society Activation plan of Internet of Things(IoT)", KIET Monthly Industrial Economics Issue paper, (2014) February 20.

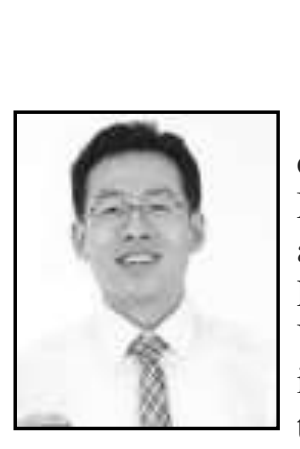

\section{Authors}

Yong-kyu Lee, he holds a bachelor's degree in information and communications from Induk University. He graduated from Myongji University with Master of Science (MS) degree, and is an expert in 3D printing and Deep Learning. Currently, he is a Ph.D. student on Graduate School of Venture at Hoseo University, $\mathrm{He}$ is a specialist in technical field of the 4th industrial revolution, technical guidance, lecture and research in the field. 


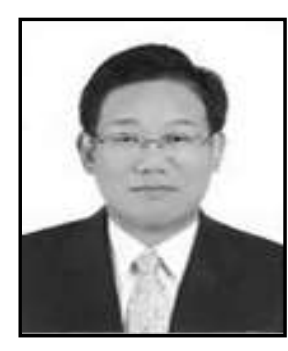

Dea-woo Park, he is an Associate Professor in the Hoseo Graduate School of Venture at Hoseo University in South Korea. Professor Park researches of Cybersecurity, Hacking Forensic, Convergence of Information Communication Technology in HFICT Lab at Hoseo Graduate School, Professor Park received the B.S. degree in computer science from the Soongsil University in 1995. And he received the M.S. degree in 1998. He received the Ph.D. degree from the computer science department of the Soongsil University in 2004. He has also been appointed, Secretary General of Forum of National Cybersecurity Policy, and Chair of Korea Information Security Forum. Professor Park has been appointed ViceChairman of Korea Institute of Information Security \& Cryptology, Korea Information and Communications Society, Korea Digital 